\title{
Grammatical Swarm based Segmentation Methodology for Lesion Segmentation in Brain MRI
}

\author{
Tapas Si \\ Dept. of ECE \\ National Institute of Technology Durgapur \\ West Bengal, India
}

\author{
Arunava De \\ Dept. of IT \\ Dr. B.C. Roy Engineering College \\ Duragpur, West Bengal, India
}

\author{
Anup Kumar Bhattacharjee \\ Dept. of ECE \\ National Institute of Technology Durgapur \\ West Bengal, India
}

\begin{abstract}
This paper presents Grammatical Swarm based segmentation methodology for lesion detection in brain's magnetic resonance image. In the proposed methodology, images are denoised using median filter at the outset. Secondly, images are segmented using Grammatical Swarm based hard-clustering technique. Finally, lesions are extracted from the segmented images. The proposed methodology is applied on six Axial-T2 magnetic resonance images and compared with Particle Swarm Optimizer, K-Means and FCM based segmentation methods using quantitative performance measurement index. The experimental results show that the proposed methodology statistically outperforms other methods.
\end{abstract}

\section{General Terms:}

Medical Image Processing, Machine Learning, Swarm Intelligence

\section{Keywords}

Brain, Magnetic resonance image, Lesion, Segmentation, Clustering, Grammatical swarm, Particle swarm optimizer

\section{INTRODUCTION}

\subsection{Background}

In medical diagnosis, mutlimodal Magnetic Resonance Imaging (MRI) [1, 2] segmentation is an important image analysis task. Segmentation of multimodal MRI is now very important in detection of lesion in the brain. Magnetic resonance imaging provides detailed information about brain lesions, tumor anatomy, vascular supply and cellular structure. For the effective diagnosis, treatment and monitoring of the brain disease, it is now an important tool [1]. MR images of brain can have maximum seven classes or objects [5]:(i) background, (ii) cerebrospinal fluid(CSF), (iii) white matter, (iv) gray matter,(v) bone, (vi) scalp and (vii) lesion. There are different types of modalities of brain MRI of the same patient: (a) T1-Weighted (b) T2-Weighted (c) Proton density ( $\rho$-weighted) (d)Fluid-attenuated inversion recovery (FLAIR) (e)diffusion-weighted(DW) (f) Perfusion-weighted (PW) [2]. In this article, only T2-Weighted images are used for segmentation because this type of modality has the intrinsically higher soft tissue contrast resolution. Segmentation is a process to partition the image into different regions or segments or class. The main difficulties in
MRI segmentation are (a) noise (b) the bias field (intensity inhomogeneity i.e. smooth intensity change inside originally homogeneous regions) and (c) the partial volume effect i.e. a voxel contributes in multiple tissues [6, 7]. During past several years, a lot of contributions has been made in segmentation as well as detection of lesion or tumor in brain MRI and some of these are discussed in the next subsequent section.

\subsection{Related Works}

Brain MRI segmentation is to divide the image into different class of tissues as well as to detect lesion. W. M. Wells et al. [8] proposed an adaptive segmentation of MRI images and this method used the knowledge of tissue intensity properties and intensity inhomogeneities to correct and segment the MRI image. ExpectationMaximization (EM) algorithm was used to obtain the bias field. A knowledge-based technique for automatic segmentation of brain MRI was presented in article [9]. First, Fuzzy C-Means (FCM) clustering algorithm is used to segment the image in this method. An expert system was used with initial FCM segmentation to detect normal or abnormal slice of MRI. S. Saha et al. [10] proposed an automatic segmentation technique of multispectral magnetic resonance image of the brain using new fuzzy point symmetry based genetic clustering technique. Real-coded variable string length genetic fuzzy clustering technique (Fuzzy-VGAPS) was used to evolve the number of clusters present in the Multiple Sclerosis MRI data set automatically. M. Y. Siyal et al. [11] proposed an intelligent modified fuzzy c-means based algorithm for bias (or intensity inhomogeneity) estimation and segmentation of brain MRI. A. Dasgupta [12] segmented the brain MRI for lesion detection using a modified Fuzzy C-Means algorithm which can filter the image at the time of segmentation of noisy image. A. De et al. [13] proposed masking based segmentation of diseased MRI Images. An entropy based maximization using Particle Swarm Optimizer was used to select the suitable threshold value for brain MRI segmentation to separate the lesions from healthy tissue cells and a variable mask is used to de-noise the image. A. De et al. [3, 4] used hybrid particle swarm optimization with wavelet mutation based segmentation for brain MRI. In these methods entropy based maximization is used to select proper threshold values for segmentation of the images. J. Alirezaie et al. [5] used Back-Propagation neural network and Learning Vector Quantization neural network to segment the brain MR images. In the year 2013, S. Sindhumol et al. [14] proposed an automated brain tissue classification by multi- 
signal wavelet decomposition and independent component analysis. In this method, a multi-signal wavelet analysis is applied on input multispectral data. Signals are reconstructed from detail coefficients were used in conjunction with original input signals to do Independent Component Analysis (ICA). Fuzzy C-Means (FCM) clustering was performed on generated results for segmentation. Y. Kong et al. [15] proposed an information theoretic discriminative segmentation method with supervoxel level feature extraction for brain MR images. A. Islam et al. [16] introduced the detection and segmentation of brain tumors using multifractal texture estimation. T. Wang et al. [17] proposed a fluid vector flow (FVF) active contour model to tumor segmentation in brain MRI. M. Huang et al. [18] proposed a local independent projection-based classification (LIPC) method using learning a softmax regression model for brain tumor segmentation in MRI. E.S. A. E. Dahshan et al. [19] made a State-of-the-Art review works on CAD systems developed during the year 2005-2015 and also proposed a hybrid intelligent machine learning technique in which they used principle component analysis (PCA) for reducing the Wavelet features and Feedforward multilayer neural network (FFNN) for classification for automatic detection of brain tumor. M.S. Yang et al. [20| presented fuzzy-soft LVQ neural network in segmenting brain MR images. $\mathrm{N}$. Zhang et al. [21] proposed tumor segmentation in brain's MRI using Support Vector Machine (SVM) with feature selection in kernel space. Grammatical Swarm based clustering algorithm (CGS) is applied on Magnetic Resonance images and lesions are separated from the healthy brain matters in [22].

This article is an extension of the work done by [22]. In this improved segmentation methodology, images are de-noised using $3 \times 3$ median filter at first. Second, an clustering technique with Grammatical Swarm (CGS) is used to segment the de-noised images. Finally, the lesions are extracted from the segmented images by thresholding and connected component algorithm. In the article [22], the CGS algorithm was applied on only two Axial-T2 MR images and was not compared previously with any existing clustering methods. In this paper, the proposed methodology is applied on six Axial-T2 MR images and a comparative study is done with PSO [23], K-Means [29] and FCM [30] based segmentation methods with both qualitative and quantitative measurement index (i.e Dunn Index [24, 25]). Finally, statistical significance in the performances of the methods has been tested. The proposed methodology statistically outperforms other methods.

\subsection{Organization of this article}

The article is organized as follows:

Section 2 discusses the materials and methods. Experimental setup is detailed in section 3 , results and Analysis is given in section 4 and finally section 5 deals with conclusions and future enhancements.

\section{MATERIALS \& METHODS}

The proposed segmentation methodology has five steps. These steps are given below:

(1) MR Image data acquisition.

(2) Denoising using $3 \times 3$ median filter.

(3) Segmentation using Grammatical Swarm based hardclustering technique.

(4) Extraction of lesions from segmented MR images.

(5) Area calculation of lesions.

The flowchart of the proposed methodology is given in Fig. 1 The various steps are discussed in detail in the next.

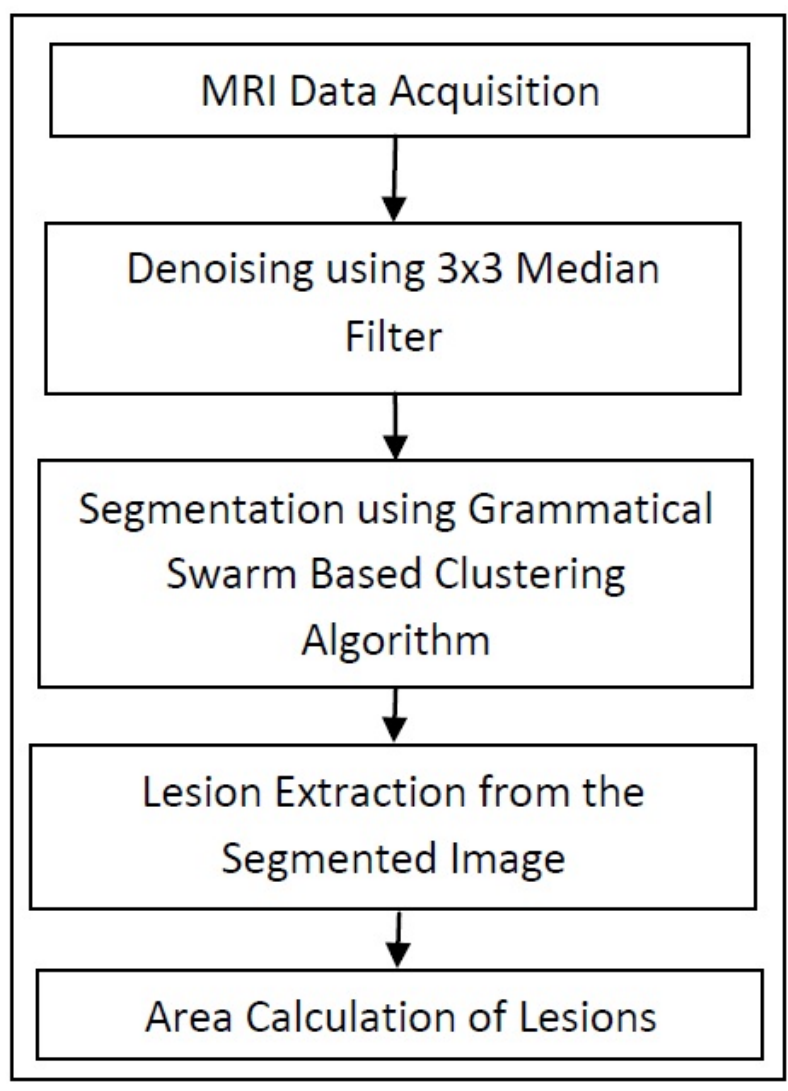

Fig. 1. Flowchart of the proposed method.

\subsection{MRI Data Acquisition}

Four Axial-T2 MRI images of human brain have been used for the application purpose. All the images are generated by 1.5-T GE Medical MRI machine. The slice thickness is $5.0 \mathrm{~mm}$, the gap between consecutive slices is $1.5 \mathrm{~mm}$. The resolution of each MR image is $256 \times 256$.

\subsection{Denoising}

Segmentation and lesion detection process faces problems due to presence of noise across the images. The noises are removed from images using median filter with window size $3 \times 3$. The median is calculated by first sorting all the pixel values from the window (i.e pattern of neighbors) into numerical order, and then replacing the pixel being considered with the median pixel value.

\subsection{Segmentation Method}

After denoising of the MR images, partitional clustering technique is used to segment the images. Clustering is an unsupervised learning to explore the unknown nature of data through the separation of a finite dataset, with or without a priori knowledge, into a finite and discrete set of hidden data structures [27, 28]. Iterative Kmeans algorithm [29] is a widely used partitional clustering algorithm. The objective of K-means algorithm is to minimize of intracluster spread (ICS) for $\mathcal{K}$ number of clusters and it is expressed as 
following:

$$
I C S=\sum_{k=1}^{\mathcal{K}} \sum_{x_{i} \in \mathcal{C}_{i}}\left\|x_{i}-m_{i}\right\|^{2}
$$

where $x_{i}$ is the pattern in $\mathcal{C}_{i}$ cluster and $m_{i}$ is the mean of the patterns in the same cluster and $\|$.$\| is the Euclidean norm. The$ $\mathrm{K}$-means algorithm starts with randomly selected $\mathcal{K}$ cluster centers. Each pattern $x_{i}$ in the data set is then assigned to the closest cluster. The centers are updated by the mean of the associated patterns in the cluster. The algorithm is run until the maximum number of iterations are reached or there is no improvement during some successive iterations. Though $\mathcal{K}$-means algorithm is very easy to implement, it's performance is data dependent and it is heavily dependent on the initial cluster's centers. This results $\mathcal{K}$-means to converge to suboptimal solutions.

The Swarm Intelligent or meta-heuristic algorithms [31] like Particle Swarm Optimizer (PSO) [23], Artificial Bee Colony (ABC) [32] were applied to the partitional clustering problem and outperformed $\mathcal{K}$-means algorithm in MR image classification or segmentation. PSO has quick convergence speed but it often suffers from premature convergence due to lacks in diversity. In this paper, Grammatical Swarm based partitional clustering (CGS) algorithm is used in segmentation and Grammatical Swarm based clustering algorithm performs better than clustering with PSO [23] because GS has more exploration ability in the search space than PSO. Grammatical Swarm based clustering algorithm is discussed in next.

2.3.1 Grammatical Swarm Based Clustering Algorithm. T. Si et al. [22] proposed Grammatical Swarm based clustering algorithm (CGS) in brain MRI segmentation. In this method, after getting denoised image using DWT based soft-thresholding technique, Grammatical Swarm based clustering algorithm is used for segmentation. Generally, Grammatical Swarm is a variant of Grammatical Evolution which is used to generate computer programs in any arbitrary language. Grammatical Swarm uses Particle Swarm Optimization as a searching algorithm in genotype-to-phenotype mapping process. Particle Swarm Optimization is discussed in next.

2.3.1.1 Particle Swarm Optimization. Particle swarm optimization (PSO) [33] is a Swarm Intelligent algorithm used for function optimization. Each individual in PSO is termed as a particle and a set of particles is termed as swarm. The current position $y_{i}$ of $i^{t h}$ particle is represented as $<y_{i 1}, y_{i 2}, y_{i 3}, \ldots, y_{i d}>$ where $d$ is the dimension of particle's position. Each particle stores its personal best $y_{i}^{p}$ found so far. The best of all personal best solution is known as global best $y^{g}$ of the swarm. Each particle is accelerated by its velocity $v_{i}$ updated by the following equation:

$$
\begin{aligned}
v_{i}(t+1)=w \times v_{i}(t)+c_{1} & \times r_{1} \times\left(y_{i}^{p}(t)-y_{i}(t)\right) \\
& +c_{2} \times r_{2} \times\left(y^{g}(t)-y_{i}(t)\right)
\end{aligned}
$$

and position is updated as following:

$$
y_{i}(t+1)=y_{i}(t)+v_{i}(t+1)
$$

In Eq. 22, $w \in(0,1)$ is the inertia weight, $c_{1}$ and $c_{2}$ are the personal cognizance and social cognizance respectively. $r_{1}$ and $r_{2}$ are two uniformly distributed random number generated in the range $(0,1)$.

Inertia weight is linearly decreased with time in the range $\left(w_{\min }, w_{\max }\right)$ using the following equation [34].

$$
w=w_{\max }-\left(w_{\max }-w_{\min }\right) \times\left(\frac{t}{t_{\max }}\right)
$$

2.3.1.2 Backus-Naur Form of CFG. In Grammatical Swarm based partitional clustering algorithm, the solutions i.e cluster's centroids are generated from the particle position by means of BNF of Context-Free Grammar and real valued particle's position is rounded to nearest integer to form codons in the range $[0,255]$. The probable solutions are integer values in the range $[0,255]$ because the image has gray values from 0 to 255 . An integer value in the range $[0,255]$ can be generated using the following grammar:

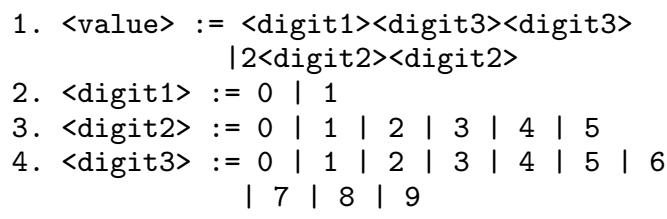

The value in the range $[0,199]$ can be generated by starting the derivation with production rule $<$ value $>:=$ $<$ digit $1><$ digit $3><$ digit $3>$. The value in the range $[200,255]$ can be generated by starting the derivation with production rule $<$ value $>:=2<$ digit $2><$ digit $2>$.

2.3.1.3 Genotype-to-Phenotype Mapping. Only four codons in genotype are needed to generate one integer value. If the number of cluster is $\mathcal{K}$ then the dimension of the position of the particle in GS is $D=4 \mathcal{K}$. If any brain MR image contains the seven classes [5], then maximum number of clusters will be 7 . For this application, the maximum dimension in GS can be $4 \times 7=28$.

\section{\begin{tabular}{|l|l|l|l|}
\hline 190 & 157 & 160 & 198 \\
\hline
\end{tabular}}

Fig. 2. An example of a part of genotype

A mapping process is used to map from integer-valued codon to the rule number in the derivation of centers using BNF of CFG by the following ways:

rule $=($ codon integer value) MOD (number of rules for the current non-terminal)

If the current non-terminal $<$ value $>$ is in the derivation process, then the rule number is generated by the following rule:

rule number $=(190 \bmod 2)=0$

$<$ value $>$ will be replaced by $(<$ digit $1><$ digit $3><$ digit $3>$ ).

A complete derivation from genotype in Fig. 2 is given as following:

$$
\begin{aligned}
<\text { value }> & :=<\text { digit } 1><\text { digit } 3><\text { digit } 3> & & (190 \bmod 2)=0 \\
& :=1<\text { digit } 3><\text { digit } 3> & & (157 \bmod 2)=1 \\
& :=10<\text { digit } 3> & & (160 \bmod 10)=0 \\
& :=108 & & (198 \bmod 10)=8
\end{aligned}
$$

2.3.1.4 Pixel Clustering. After mapping cluster's centers $m_{k}(\forall k \in[1, \mathcal{K}])$ from the particle's position $y_{i}$, the euclidean distance of a pixel gray value $x_{n}$ is measured by the following equation:

$$
\mathcal{D}_{n k}=\left[\sum_{l=1}^{d}\left\|x_{n l}-m_{k l}\right\|^{2}\right]^{\frac{1}{2}}
$$

The closest cluster number $k$ is assigned to pixel $x_{n}$ using the following equation:

$$
k=\min _{\forall k \in\{1,2, \ldots, K\}}\left\{\mathcal{D}_{n k}\right\}
$$


2.3.1.5 Fitness Measure using Dunn Index. In the proposed method, a quantitative measurement index called as Dunn Index [24 25] is used to measure the performance of clustering algorithm. Dunn index is defined as following:

$$
\mathcal{D I}=\min _{i}\left\{\min _{i \neq j}\left\{\frac{d\left(m_{i}, m_{j}\right)}{\max _{k} \mathcal{S}_{q}\left(m_{k}\right)}\right\}\right\}, \quad 1 \leq i, j, k \leq \mathcal{K}
$$

$d\left(m_{i}, m_{j}\right)$ is the inter cluster distance between clusters $\mathcal{C}_{i}$ and $\mathcal{C}_{j}$ where $m_{i}$ and $m_{j}$ are the centers respectively. $\mathcal{S}_{q}\left(m_{i}\right)$ is the standard deviation of intra-cluster distances in $i^{\text {th }}$ cluster and it is defined by the following equation:

$$
\mathcal{S}_{q}\left(m_{i}\right)=\left[\frac{1}{\mathcal{N}_{i}} \sum_{x \in \mathcal{C}_{i}}\left|x-m_{i}\right|^{q}\right]^{\frac{1}{q}}
$$

where $q=2$. The higher value of Dunn Index indicates better clustering. But, PSO minimizes the objective function by default. Therefore, fitness of particles are assigned as follows: fit $_{i}=$ $-\mathcal{D I}_{i}$. The Grammatical Swarm based clustering (CGS) Algorithm is described in Table 1.

\begin{tabular}{|c|c|}
\hline & Algorithm:CGS \\
\hline 1. & Initialize the population $y$ of population size $\mathcal{N}$ \\
\hline 2. & Initialize the velocity $v$ \\
\hline 3. & Decode the cluster's centroids $m_{k}$ from the $y_{i}$ \\
\hline 4. & $\begin{array}{l}\text { Calculate the distances } \mathcal{D}_{n k} \text { for each pixel } x_{n} \text { from each cluster's } \\
\text { centroid using Eq. } 5 \text { and assign the closest cluster using Eq. } 6 \text { : }\end{array}$ \\
\hline 5. & Calculate the fitness $f i t_{i}$ of all particles \\
\hline 6. & Calculate pbest and gbest. \\
\hline 7. & $g=1$ \\
\hline 8. & while $g \leq G E N_{\max }$ \\
\hline 9. & for $i=1: \mathcal{N}$ \\
\hline 10 . & Update velocity $v_{i}$ and position $y_{i}$ using Eq. 2) and Eq. (3) \\
\hline 11. & Do the steps $3,4 \& 5$ \\
\hline 12. & Calculate new fitness $f i t_{i}$ \\
\hline 13. & Update pbest and gbest \\
\hline 14. & End for \\
\hline 15 . & $g=g+1$ \\
\hline 15 . & End while \\
\hline
\end{tabular}

\section{Table 1. CGS Algorithm}

\subsection{Lesion Extraction}

After segmentation process, thresholding is done to get regions of interest (ROI) and the highest intensity value of the cluster centers is used as threshold. A connected component labelling algorithm with neighbourhood size 4 is used to extract lesions from ROIs.

\subsection{Area Calculation}

After extraction, the area of lesions is calculated in terms of total number of pixels in the lesioned images.

\section{EXPERIMENTAL SETUP}

\subsection{Parameter Settings}

The parameters of GS and PSO [23] algorithms are given in Table 3.1 The population size and maximum number of iterations are common in GS, PSO and these are set in such a way that all the methods can evaluate maximum 3000 function evaluations. Therefore, K-means and FCM methods are also ran with 3000 function evaluations.

Table 2. Parameter Settings

\begin{tabular}{|c|c|c|}
\hline Parameters & GS & PSO \\
\hline Dimension $(d)$ & $4 . \mathcal{K}$ & $\mathcal{K}$ \\
\hline Population Size $(\mathcal{N})$ & 30 & 30 \\
\hline$V_{\max }$ & $0.5 \times\left(X_{\max }-X_{\min }\right)$ & 4 \\
\hline$c_{1}=c_{2}$ & 1.49445 & 1.49445 \\
\hline$w_{\max }, w_{\min }$ & $0.9,0.4$ & - \\
\hline$w$ & - & 0.72 \\
\hline$w_{1}, w_{2}$ & - & $0.5,0.5$ \\
\hline Maximum number of iterations & 100 & 100 \\
\hline
\end{tabular}

\subsection{PC Configuration}

(1) Operating System: Windows 7

(2) CPU: AMD FX -8150 Eight-Core $3.6 \mathrm{GHz}$

(3) RAM: 16 GB

(4) Software: Matlab 2010b

\section{RESULTS AND ANALYSIS}

The proposed methodology is applied with cluster number $\mathcal{K}=4$ on six Axial-T2 MR images given in the first column of Fig. 3. The PSO, K-Means and FCM based methods are also applied on the same set of images. The qualitative results in the form of segmented images and extracted lesioned images with the best run are given in Fig. 3 and Fig. 4 respectively. To measure the performance of the methods, quantitative measure Dunn-Index is used. The mean and standard deviation (in parenthesis) of Dunn-Index over 30 independent runs are given in Table 3 The area of lesion in brain is calculated in terms of total number of pixels in lesioned images and it is given in Table 4

From Fig. 3 it can be visually determined that the proposed method performs better than PSO, K-Means and FCM based methods. It can be observed that PSO gets trapped into local optima for most of the images. Though K-Means performs better than PSO for most of the images, it also suffers from local optima problem. FCM based method performs well only for $3^{\text {rd }}$ image (in the first column of Fig. 37. From Fig. 4 it is seen that proposed method performs better than other methods in lesion detection.

In Table 3 the mean Dunn-Index values of the proposed method over 30 independent runs are highest for all the images. Therefore, GS based clustering technique performs better segmentation of the MR images than other methods. To test the overall statistical significance of the differences in the performances of the proposed method with the other methods, Wilcoxon sign rank test [35] has been done using the mean Dunn-Index values of 30 independent runs for all images and the test results are given in Table 5 From the Table 5 it is seen that the proposed method statistically performs better than all other methods with significance level $\alpha=0.05$. In Table 3 the standard deviations of Dunn-Index of the proposed method over 30 independent runs are lower for all the images than PSO and K-Means based methods. Therefore, GS based clustering technique is more robust than PSO and K-Means based methods. FCM based method is more robust than the proposed method but it fails to deliver good quality of solutions. From this quantitative analysis, it can be said that the proposed method performs better than other methods with higher robustness. The calculated areas of 
extracted lesions obtained using the proposed method from the best run in Table 4 are more accurate than other methods.

GS algorithm provides more exploration in the search space results in better quality of solutions whereas PSO, K-Means and FCM prematurely converge to sub-optimal solutions. The proposed methodology has also few limitations. Due to similarities in the intensity of the pixels, other parts of healthy tissues are also segmented with the lesions. These parts are well separated by connected component algorithm. If the healthy tissues are connected with the lesion then it cannot be segmented by connected component algorithm. Another limitation of the proposed methodology is that the user has to give cluster number explicitly.

The above analysis of both qualitative and quantitative results proves that the proposed method performs better than other methods.

Table 3. Mean and standard deviation of Dunn-Index over 30 separate runs (values in bold face indicates better)

\begin{tabular}{|l|l|l|l|l|}
\hline MRI\# & GS & PSO & K-Means & FCM \\
\hline 1 & $\mathbf{1 5 . 7 0 ( 0 . 8 5 6 4 )}$ & $9.99(2.5949)$ & $10.74(2.2056)$ & $5.60(1.42 \mathrm{e}-6)$ \\
\hline 2 & $\mathbf{1 6 . 8 2 ( 1 . 0 3 4 6 )}$ & $10.40(2.1913)$ & $12.79(4.4809)$ & $5.72(8.00 \mathrm{e}-6)$ \\
\hline 3 & $\mathbf{1 5 . 9 6 ( 0 . 6 0 9 6 )}$ & $9.70(2.9899)$ & $11.62(2.4006)$ & $10.91(3.42 \mathrm{e}-6)$ \\
\hline 4 & $\mathbf{1 6 . 2 0}(\mathbf{0 . 7 2 5 2})$ & $9.74(2.2719)$ & $12.24(1.7329)$ & $6.14(3.67 \mathrm{e}-7)$ \\
\hline 5 & $\mathbf{1 6 . 2 5}(\mathbf{0 . 7 9 7 7})$ & $10.75(1.8014)$ & $8.22(0.1393)$ & $7.98(1.4650 \mathrm{e}-5)$ \\
\hline 6 & $\mathbf{1 5 . 2 8}(\mathbf{0 . 5 6 1 5})$ & $10.25(2.2123)$ & $8.98(0.0649)$ & $8.67(6.7805 \mathrm{e}-6)$ \\
\hline
\end{tabular}

Table 4. Areas of extracted lesions (with best run)

\begin{tabular}{|c|r|r|r|r|}
\hline MRI\# & GS & PSO & K-Means & FCM \\
\hline 1 & 349 & 238 & 516 & 2166 \\
\hline 2 & 655 & 660 & 722 & 3424 \\
\hline 3 & 816 & 829 & 914 & 933 \\
\hline 4 & 592 & 281 & 819 & 1479 \\
\hline 5 & 509 & 450 & 2431 & 2263 \\
\hline 6 & 238 & 45 & 1841 & 1908 \\
\hline
\end{tabular}

Table 5. Wilcoxon Signed Ranks Test Statistics using mean Dunn-Index values over 30 independent runs. $R^{+}$: sum of positive ranks, $R^{-}$: sum of negative ranks

\begin{tabular}{|c|l|c|c|c|c|}
\hline Sl. No. & Comparison & $R^{+}$ & $R^{-}$ & $Z$ & $p(2-$ tailed $)$ \\
\hline 1 & GS $v$ PSO & 21 & 0 & -2.201 & 0.028 \\
\hline 2 & GS $v$ K-means & 21 & 0 & -2.201 & 0.028 \\
\hline 3 & GS $v$ FCM & 21 & 0 & -2.201 & 0.028 \\
\hline
\end{tabular}

\section{CONCLUSIONS}

This paper proposes Grammatical Swarm based segmentation methodology for lesion detection in MR image of brain. First, MR images are denoised using median filter. Grammatical Swarm based hard-clustering algorithm is used to segment the de-noised images. Thresholding is performed to get region of interest containing lesion. Finally, the lesions are extracted from the the region of interests using connected component labelling algorithm. The proposed methodology is applied on six Axial-T2 MR images. A comparative study is made with PSO, K-Means and FCM based methods. Both qualitative and quantitative analysis of the experimental result demonstrates that the proposed segmentation methodology performs better than other methods. The proposed segmentation methodology can be further improved by removing intensity inhomogeneities in the MR images. In the proposed methodology, clustering of images are carried out using predefined cluster numbers given explicitly. Automatic clustering with Grammatical Swarm will be developed in future to detect the number of clusters automatically in the MR images.

\section{REFERENCES}

[1] Paul M. Parizel, Luc van den Hauwe, Frank De Belder, J. Van Goethem , CarolinVenstermans, Rodrigo Salgado, Maurits Voormolen, and Wim Van Hecke: Magnetic Resonance Imaging of the Brain, P. Reimer et al. (eds.): Clinical MR Imaging, Springer-Verlag Berlin Heidelberg,2010.

[2] L. Tonarelli: Magnetic Resonance Imaging of Brain Tumor, CEwebsource.com, 2013.

[3] A. De, A. K. Bhattacharjee and C. K. Chanda and B. Maji: Hybrid Particle Swarm Optimization With Wavelet Mutation Based Segmentation and Progressive Transmission Technique For MRI Images, International Journal of Innovative Computing, Information and Control, Vol. 8,No. 7(B), pp. 51795197, 2012.

[4] A. De, A. K. Bhattacharjee and C. K. Chanda and B. Maji: Entropy Maximization Based Segmentation, Transmission and Wavelet Fusion of MRI Images, International Journal of Hybrid Intelligent Systems, IO Press, Vol. 10, pp. 57-69, 2013.

[5] J. Alirezaie, M. E. Jernigan, and C. Nahmias: Neural Network based Segmentation of Magnetic Resonance Images of the Brain, IEEE Transactions on Nuclear Science, Vol. 44, Issue 2, pp. 194-198, 1997.

[6] M. A. Balafar, A. R. Ramli, M. I. Saripan and S. Mashohor: Review of brain MRI image segmentation methods, Artif. Intell. Rev.,Vol.33 pp. 261-274, 2010.

[7] M. A. Balafar, A. R. Ramli, and S. Mashohor: A new method for MR grayscale inhomogeneity correction, Artif. Intell. Rev.,Vol.34 pp. 195-204, 2010.

[8] W. M. Wells et al.: Adaptive Segmentation of MRI Data, IEEE Transaction on Medical Imaging, Vol. 15, No. 4, pp. 429-442, 1996.

[9] M. C. Clark, L. O. Hall, D. B. Goldgof, R. Velthuizen, F. R. Murtagh, and M. S. Silbiger: Automatic Tumor Segmentation Using Knowledge-Based Techniques, IEEE Transaction on Medical Imaging, Vol.17, No. 2 pp. 187-201, 1998.

[10] S. Saha and S. Bandyopadhyay, MRI Brain Image Segmentation by Fuzzy Symmetry Based Genetic Clustering Technique, IEEE Congress on Evolutionary Computation, Singapore pp. 4417-4424, 2007.

[11] M.Y. Siyal and L. Yu: An intelligent modified fuzzy c-means based algorithm for bias estimation and segmentation of brain MRI, Pattern Recognition Letters, 26, pp. 2052-2062, 2005.

[12] A. Dasgupta: Demarcation of Brain Tumor Using Modified Fuzzy CMeans, International Journal of Engineering Research and Applications, Vol. 2, Issue. 4, pp. 529-533, 2012.

[13] A. De, R. L. Das, A. K. Bhattacharjee and D. Sharma: Masking based Segmentation of Diseased MRI Images, IEEE Inter- 
national Conference on Information Science and Applications (ICISA), Seol, South Korea pp. 1-7, 2010.

[14] S. Sindhumol, Anil Kumar and Balakrishnan Kannan, Automated Brain Tissue Classification by Multisignal Wavelet Decomposition and Independent Component Analysis, ISRN Biomedical Imaging, Hindawi Publishing Corporation, Volume 2013, pp.1-10.

[15] Y. Kong, Y. Deng, and Q. Dai, Discriminative Clustering and Feature Selection for Brain MRI Segmentation, IEEE Signal Processing Letters, Vol. 22, No. 5(2015), 573-577.

[16] A. Islam, S. M. S. Reza, and K. M. Iftekharuddin, Multifractal Texture Estimation for Detection and Segmentation of Brain Tumors, IEEE Transactions on Biomedical Engineering, Vol. 60, No. 11(2013), 3204-3215.

[17] T. Wang, I. Cheng and A. Basu, Fluid Vector Flow and Applications in Brain Tumor Segmentation, IEEE Transactions on Biomedical Engineering, Vol. 56, No. 3(2009), 781-789.

[18] M. Huang, W. Yang, Y. Wu, J. Jiang, W. Chen and Q. Feng, Brain Tumor Segmentation Based on Local Independent Projection-Based Classification, IEEE Transactions on Medical Imaging, Vol. 61, No. 10, 2014, pp. 2633-2645.

[19] E.S. A. E. Dahshan, H.M. Mohsen, K. Revett, A.B. M. Salem, Computer-aided diagnosis of human brain tumor through MRI: A survey and a new algorithm, Expert Systems with Applications 41 (2014), 5526-5545.

[20] M.S. Yang, K.C.R Lin, H.C. Liu and J.F. Lirng, Magnetic resonance imaging segmentation techniques using batch-type learning vector quantization algorithms, Magnetic Resonance Imaging 25(2007),Elsevier, 265-277

[21] N. Zhang, S. Ruan,S. Lebonvallet, Q. Liao and Y. Zhu, Kernel feature selection to fuse multi-spectral MRI images for brain tumor segmentation, Computer Vision and Image Understanding 115 (2011), pp. 256-269

[22] T. Si, A. De and A. K. Bhattacharjee: Brain MRI Segmentation for Tumor Detection using Grammatical Swarm Based Clustering Algorithm , IEEE International Conference on Circuit, Power and Computing Technologies, Tamilnadu, India, pp.1196-1201, 2014.

[23] M. G.H. Omran, A. Salman and A. P. Engelbrecht: Image classification using Particle Swarm Optimization, Proceedings of the 4th Asia-Pacific conference on simulated evolution and learning, Volume 1, Pages 18-22.

[24] J. C. Bezdek and N. R. Pal: Some New Indexes for Cluster Validity, IEEE Transactions on System, Man, and Cybernetics, Part B, Vol. 28, 301-315, 2007.

[25] P. Maji and S. K. Pal: Rough Set Based Generalized Fuzzy CMeans Algorithm and Quantitative Indices, IEEE Trans. On Systems, Man, and Cybernetics-Part B: Cybernetics, Vol. 37, No. 6, pp. 1529-1540, 2007.

[26] M. O'Neill, A. Brabazon: Grammatical Swarm: The Generation of Programs by Social Programming, Natural Computing 5(4),pp. 443-462.

[27] R. Xu and D. C. Wunsch: Survey of Clustering Algorithms, IEEE Transaction On Neural Networks, Vol. 16, No. 3, pp. 645-678, 2005.

[28] R. Xu and D. C. Wunsch: Clustering Algorithms in Biomedical Research: A Review, IEEE Reviews In Biomedical Engineering, pp. 120-154, 2010.
[29] J. MacQueen: Some methods for classification and analysis of multivariate observations, in Proc. 5th Berkeley Symp. Math. Stat. Probability, 1967, pp. 281-297.

[30] J. C. Bezdek, R. Ehrlich, and W. Full: FCM: The Fuzzy c-Means Clustering Algorithm, Computers \& Geosciences,U.S.A, Vol. 10, No. 2-3, pp. 191-203(1984).

[31] S. J. Nanda and G. Panda: A survey on nature inspired metaheuristic algorithms for partitional clustering, Swarm and Evolutionary Computation, Vol. 16, pp. 1-18, 2014

[32] E. Hancer, C. Ozturk and D. Karaboga: Extraction of Brain Tumors from MRI Images with Artificial Bee Colony based Segmentation Methodology, 2013 8th IEEE International Conference on Electrical and Electronics Engineering (ELECO), pp. 516-520, 2013, DOI: 10.1109/ELECO.2013.6713896

[33] J. Kennedy and R. C. Eberhart: Particle swarm optimization, In proceedings of IEEE International Conference on Neural Networks, Piscataway, NJ. pp. 1942-1948, 1995

[34] Y. Shi and R. C. Eberhart: A modified particle swarm optimizer. In Proceedings of the IEEE Congress on Evolutionary Computation (CEC 1998), Piscataway, NJ. pp. 69-73, 1998

[35] J. Derrac, S. Garcia, D. Molina, F. Herrera, A practical tutorial on the use of nonparametric statistical tests as a methodology for comparing evolutionary and swarm intelligence algorithms, Swarm and Evolutionary Computation, 1, 3-18(2011) 

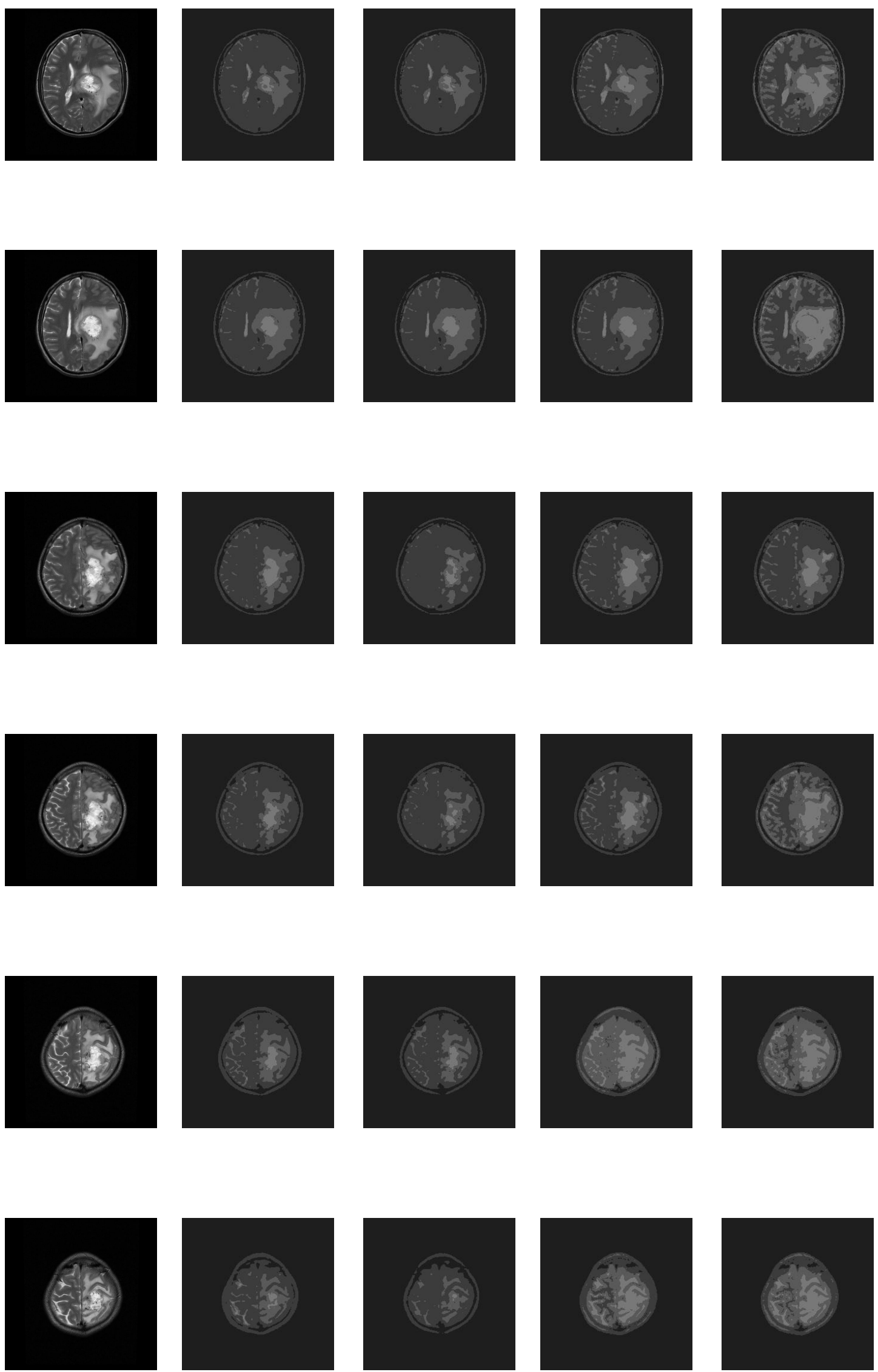

Fig. 3. $1^{\text {st }}$ column: original MR images to be segmented, $2^{\text {nd }}$ column: segmentation output of GS, $3^{\text {rd }}$ column: segmentation output of PSO, $4^{\text {th }}$ column: segmentation output of K-Means, $5^{\text {th }}$ column: segmentation output of FCM (partition become less prominent from $3^{r d}$ column to $4^{\text {th }}$ column). 


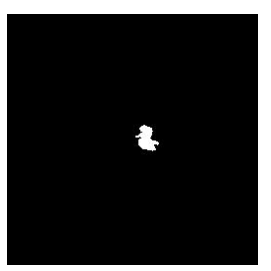

(a)

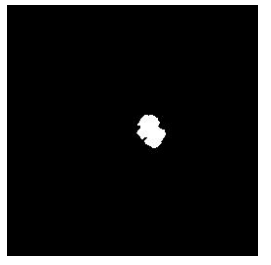

(e)

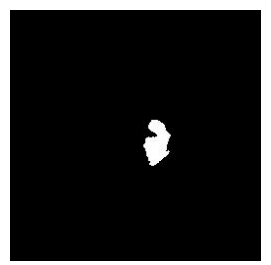

(i)

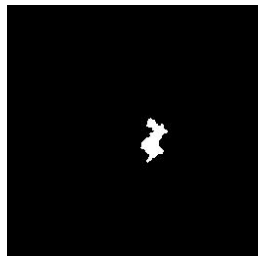

(m)

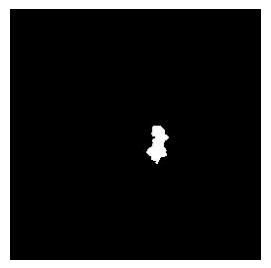

(q)

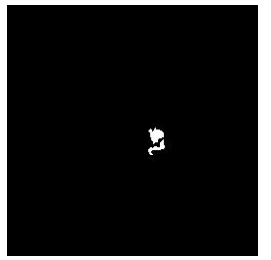

(u)

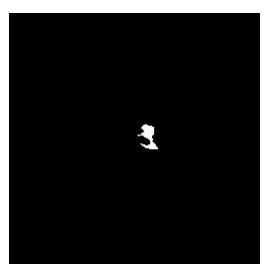

(b)

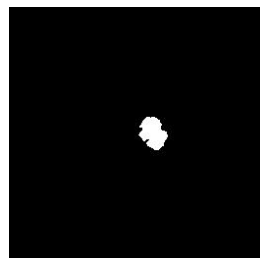

(f)
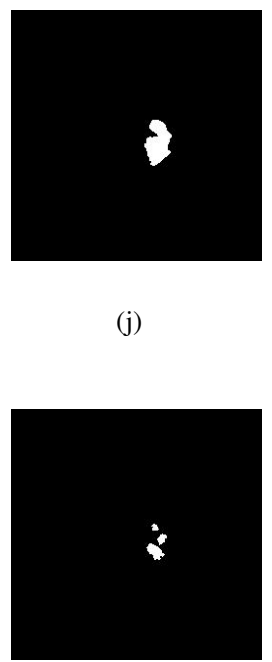

(n)

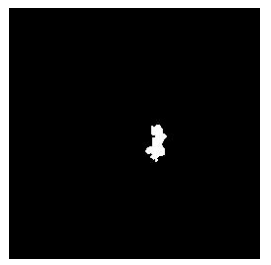

(r)

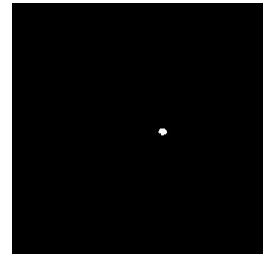

(v)

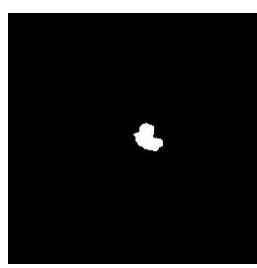

(c)

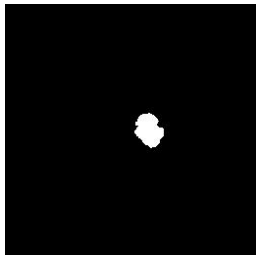

(g)

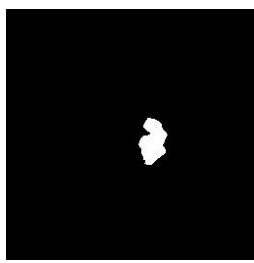

(k)

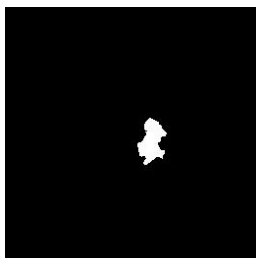

(o)

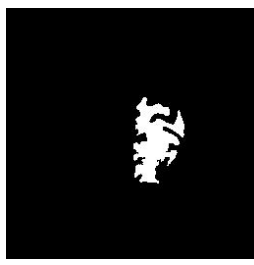

(s)

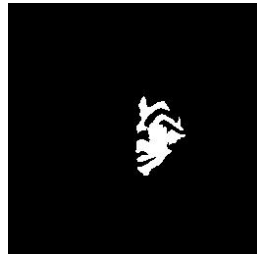

(w)

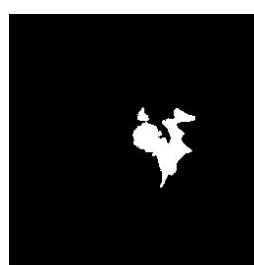

(d)

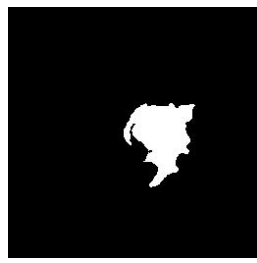

(h)

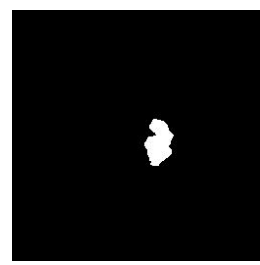

(1)

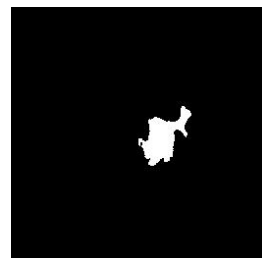

(p)

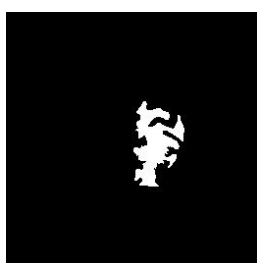

(t)

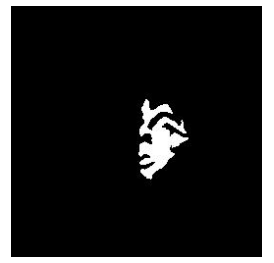

(x)

Fig. 4. Extracted lesions from segmented MR images (with best run), $1^{\text {st }}$ column: GS, $2^{\text {nd }}$ column: PSO, $3^{\text {rd }}$ column:K-Means, $4^{\text {th }}$ column:FCM. 